\title{
Agile Multicriteria Decision Analysis considering Interaction
}

\author{
ANNIBAL PARRACHO SANT’ANNA \\ Doctoral Program in Sustainable Management Systems \\ Universidade Federal Fluminense \\ Rua Passo da Pátria, 156, Niterói-RJ \\ BRAZIL
}

\begin{abstract}
An agile system of multiple criteria decision analysis is proposed here. Its main component avoids the collection of field direct information about the importance of the criteria and of the interaction between criteria. This is replaced by a derivation of Choquet capacities to the sets of criteria from the preferences they assign to the alternatives evaluated. This indirect derivation is made possible by a rule to assess importance and interaction based on a principle of preference concentration. The agile applicability of the system is enhanced by the admission of the sampling of initial assessments by pairwise trichotomic comparisons. This possibility reduces the need to precisely measure distances and the care about transitivity. Finally, an ordering of the decision rules based on the facility of explanation of their results provides agility for the final step of the decision. An example of application of the system is given by the study of a case of supplier evaluation.
\end{abstract}

Key-Words: - Decision analysis - Multicriteria - Criteria Interaction - Choquet capacity - Preference concentration - Composition of Probabilistic Preferences

Received: March 14, 2021. Revised: September 17, 2021. Accepted: October 5, 2021. Published: October 23, 2021.

\section{Introduction}

Multicriteria Decision Analysis (MCDA) [1] arises in situations where the choice is among alternatives identified by a set of attributes that serve distinct goals. The main steps of MCDA can be stated as establishing evaluation criteria that relate attributes to goals, evaluating the alternatives in terms of the selected criteria and applying some rule to combine such possibly contradictory evaluations into final scores for the alternatives.

The contradictions between the results of the application of distinct points of view may inform on the possible presence of inconsistent answers and the need to select evaluators who generate discrepant evaluations to be more carefully checked in an eventual second round of individual evaluations. The importance of the criteria, the variance in the evaluations and the interaction between the criteria take time to be evaluated by experts.

MCDA deals with evaluations usually presented as crisp numerical values, which, on the other hand, bring information about often imprecise alternatives. The evaluators can only give approximate information. Thus, the composition procedure must be sensitive to the inconsistencies and contradictions between the individual evaluations. The ability to deal with imprecise subjective information is particularly important when dealing with decisions where starting action is impending.

There are situations where there is no time to develop the consideration of these questions because an immediate decision must be taken. A health team receiving a victim of a recent injury or a military troop facing a sudden enemy attack are extreme examples of such situations. Other examples are the decision of purchasing or selling stocks in a volatile market reality or of choosing a supplier of a failing critical component.

The key issue is then to choose composition rules for the determination of aggregate rankings resistant to random distortions. Many different composition rules have been proposed to deal with these problems, each of them with its desirable properties. The first efforts to develop in a systematic way this kind of rule may be traced to [2] and [3]. Later, continuations of these efforts are found in [4-6], for instance. More recent research develops new forms of treatment of the problem combining different approaches [7-10]. In [10], TOPSIS [11] is combined with Fuzzy systems [1213]. On the other hand, [9] combines Composition 
of Probabilistic Preferences (CPP) [14] with Gray Relational Analysis [15-19].

Here an approach that takes into account interaction by means of Choquet integrals [20] is developed. Agility is particularly granted by constructing Choquet capacities without the collection of field direct information. The importance of the criteria and of the interaction between criteria is derived from their application to the alternatives evaluated applying a mechanism driven to preference concentration.

\section{Preference Concentration}

Two main features characterize an agile decision system in a multiple criteria setup: the ability to automatically deal with a great number of criteria and the ability to provide an easily understandable explanation for the choice reached. The assignment of importance to the criteria and the untangling of the interactions among the criteria constitute the most time-consuming and difficult to explain steps of the system. The principle of preference concentration [21] opens ways to pass these two steps.

The application of the principle of preference concentration to criteria evaluation is based on the idea that a criterion is considered in the decision problem if there is, in fact, a best alternative that it is able to point out. Frequently, this best alternative is hidden by the imprecision in the assessment of the attributes and there is not enough time to make clear what the correct ranking is. But, if there is such a correct ranking, higher importance should be assigned to those criteria presently pointing more clearly a best alternative. Assigning importance in such a way is expected to generate a ranking as close as possible to the correct one.

This principle, consistent with the aversion to uncertainty in the decision process, leads the decision-maker to seek the maximization of the ability to discriminate the most preferred alternative. It leads, in the case of determining the capacities of individual criteria and sets of criteria, to give greater importance to those that exhibit the greatest ability to point to an alternative as the most preferred. This corresponds to the belief that higher evaluations provide more reliable information.

Incidentally, this construction adequately considers the interactions. A preference evaluation criterion positively interacts with another, when these criteria are applied to choose the best alternative, the more so as its inclusion in addition to the others in the evaluation process increases the probability that the best alternative will be identified.

The application of this principle to criteria evaluation is similar to the maximum likelihood estimation, by assigning to the set of criteria a capacity proportional to the preference value that it assigns to its most preferred alternative.

Extracting information about preferences for criteria from available information about preferences for alternatives according to the criteria is also analogous to applying the Bayes rule, by combining new evidence to prior information. The new evidence is given by the evaluations of the different alternatives by the different criteria, while the prior information is obtained from the preliminary neutral use of the criteria.

\section{Joint preferences}

To be able to rapidly take into account a wide variety of criteria, the assessment of preferences must be simple. A simple and reliable way to discover preferences is by sampling pairwise trichotomic comparisons. Pairwise comparison grants robustness, either because of lesser difficulty of occurring errors of subjective assessment or because of the smaller effect of these possible errors.

In a trichotomic pairwise comparison, a sample of representatives of the population or of experts assesses, for each pair of alternatives, whether each alternative is more capable than each other to satisfy a desired criterion. In case of a tie, the preference is shared between the two compared candidates. Counting the number of pairs with preference for each candidate serves as the basis for calculating the preference probabilities.

If the criterion of comparison is based on the value of a numerical attribute or on a full ranking, the passage to the pairwise comparison is straightforward. Transitivity follows from that. But, if that is not the case, pairwise comparison frees from the need to preserve transitivity, as well as from the care of precisely measuring distances between alternatives.

Extracting the information about the capacity of the criteria from the pairwise comparisons generated when the criteria are applied is also simpler and more reliable than asking for direct information about the relative importance of multiple criteria. Thus, to measure the preference by a set of criteria together, counting favorable cases in the pairwise trichotomic comparison is a sound starting point. However, this count does not consider the interactions between criteria. 
To compose a reliable global preference score, it is necessary to consider the possible interactions. For this, the Choquet integral of the preference probabilities according to each criterion is here used, according to a capacity that suitably measures the relevance of each criterion and the interactions between the criteria. The measurement in terms of proportions of preference also has the advantage of bringing all evaluations to the same scale of measurement. This property, of commensurability of the probabilistic assessments, is an essential condition to make possible the combination by the Choquet integral.

There is an interaction between two criteria when the preference for an alternative by one of them increases or reduces the importance of the preference for this or any other alternative according to the other one. The same applies to groups of criteria, no matter the reason for this influence. Thus, the interaction is related to the personal aim of the decision-maker to choose the best among the available alternatives.

Following this idea, if two criteria agree to give high preference for an alternative, even if it is not the most preferred by either of them, their high joint preference for this alternative results in a high capacity for the union of the two unitary sets. In the same way more than two criteria are combined.

\section{Computational Procedures}

This section presents the practical description of the procedures for calculating the preference probabilities according to each criterion, the capacities of the criteria and, finally, the ranking of the alternatives by the Choquet integral.

The following notation will be employed: A denotes a set of alternatives, $\mathrm{N}$ denotes the number of alternatives, $i$ denotes the $i$-th alternative, $-i$ denotes the set of all alternatives other than i, J denotes a set of criteria, $\mathrm{S}$ denotes the set of all criteria, $T$ denotes the number of criteria, $j$ denotes the $\mathrm{j}$-th criterion, $\mathrm{k}_{\mathrm{j}}$ denotes the $\mathrm{k}$-th evaluator of the alternatives according to the $\mathrm{j}$-th criterion, and $\mathrm{K}_{\mathrm{j}}$ the number of evaluators according to the $\mathrm{j}$-th criterion. Other symbols will have their meaning explained across the text.

\subsection{Individual Assessments}

The probability of preference for each alternative according to each criterion is obtained from pairwise trichotomic comparisons by representative samples of the population whose preference is to be measured or from sets of experts. The results of these comparisons serve as a basis for calculating the preference probabilities according to each criterion and each evaluator.

Thus, preference probabilities $\mathrm{P}_{\mathrm{ij}}$ for each alternative $i$ according to each criterion $j$ are derived from the preference counts. This transformation from counts into probabilities is simply done by dividing the result of the count by the total number of evaluations. Let's denote by Cont $(\mathrm{j}, \mathrm{i})$ the count of preferences for the $\mathrm{i}$-th alternative according to the $\mathrm{j}$-th criterion and by $\mathrm{A}\left(\mathrm{k}_{\mathrm{j}}, \mathrm{j}, \mathrm{i}_{1}, \mathrm{i}_{2}\right)$ the result of the trichotomous comparison between alternatives $i_{1}$ and $i_{2}$ according to the $j$-th criterion by the $k_{j}$-th evaluator.

$\mathrm{A}\left(\mathrm{k}_{\mathrm{j}}, \mathrm{j}, \mathrm{i}_{1}, \mathrm{i}_{2}\right)$ has three possible values: 1 if the evaluator declares $i_{1}$ preferable to $i_{2}, 0$ if the evaluator declares $i_{2}$ preferable to $i_{1}$, and $1 / 2$ if the evaluator declares indifference between $i_{1}$ and $i_{2}$. The preference count for $i_{1}$ according to the $j$-th criterion, $\operatorname{Cont}\left(\mathrm{j}, \mathrm{i}_{1}\right)$, is the sum of the $\mathrm{A}\left(\mathrm{k}, \mathrm{j}, \mathrm{i}_{1}, \mathrm{i}_{2}\right)$ for $\mathrm{k}_{\mathrm{j}}$ varying over the set of all evaluators by the $\mathrm{j}$-th criterion and $i_{2}$ ranging over the set of all alternatives in $-\mathrm{i}$. This count is therefore the sum of the number of cases where $i_{1}$ is preferred with half the number of cases where $i_{1}$ is considered equivalent to another alternative. Finally, the probability of preference $P_{i j}$ for $i$ according to $j$ is the quotient

$\mathrm{P}_{\mathrm{ij}}=\operatorname{Cont}(\mathrm{j}, \mathrm{i}) /\left[\mathrm{K}_{\mathrm{j}} * \mathrm{~N} *(\mathrm{~N}-1) / 2\right]$.

The sum of these probabilities along $i$, for each fixed $\mathrm{j}$, is exactly 1 .

\subsection{Probabilities of being the best}

From the probabilities of preference Pij, a step into a higher concentration of preferences is the computation of the probabilities $\mathrm{B}_{\mathrm{ij}}$ of each alternative being the best according to the $\mathrm{j}$-th criterion, which is a basic step of CPP. In CPP, the initial numerical evaluation for alternative $i$ according to criterion $\mathrm{j}$ (in the present case, $\mathrm{P}_{\mathrm{ij}}$ ) determine a location parameter for a statistical distribution of possible assessments of preference. These distributions will usually be independent triangular distributions with mode in the initial numerical evaluation and extremes in possible extreme values for it, or a smoothening of such distribution (in the present case, a standard beta pert distribution). This computation is performed for each criterion. $B_{i j}$ is obtained by integrating the probability of alternative $i$ presenting a score higher than that of each other alternative regarding the $j$-th criteria. Formally,

$\mathrm{B}_{\mathrm{ij}}=\int \Pi_{\mathrm{h} \in \mathrm{i}} \operatorname{ppert}_{\mathrm{h}}(\mathrm{x}) \operatorname{dpert}_{\mathrm{i}}(\mathrm{x}) \mathrm{dx}$

where dpert ${ }_{i}$ denotes the standard beta pert density centered at $\mathrm{P}_{\mathrm{ij}}$ and ppert $_{\mathrm{h}}$ denotes the analogously 
smoothed beta pert cumulative distribution of an alternative $h$.

\subsection{Joint Assessments}

After obtaining the sets of vectors of preferences $\mathrm{P}_{\mathrm{i}}=\left(\mathrm{P}_{\mathrm{i} 1}, \ldots, \mathrm{P}_{\mathrm{iT}}\right)$ and $\mathrm{B}_{\mathrm{i}}=\left(\mathrm{B}_{\mathrm{i} 1}, \ldots, \mathrm{B}_{\mathrm{iT}}\right)$, these preferences are employed in generating the criteria's capacities that will be used in the computation of a single global score for each alternative.

A capacity on $\mathrm{S}$ is a nondecreasing function defined in the power set of $S$ with value 0 at $\phi$ and 1 at S. Capacities are measures, not necessarily additive, that express, for each subset of $S$, the importance associated with that set.

The capacity of the criteria can be obtained from assessments by experts or by any representatives of the population, but that takes time. It is harder to obtain evaluations of the importance of criteria for a given goal than evaluating alternatives according to given criteria. In contrast, it is faster to extract them from the probabilities of preference, applying the principle of preference concentration.

The principle of preference concentration leads to take into account interaction by assigning to the sets of criteria capacities proportional to the vector of maxima across the initial probabilities of preference for the alternatives according to the criteria of the set.

For each vector of preferences, three forms of derivation of the initial joint preferences by sets of criteria $\mathbf{J}$ are here employed: the first is given by the sum of the preferences according to the criteria in the set, the second is given by the maximum and the third by the optimistic progressive rule of CPP that consists in evaluating the $\mathrm{i}$-th alternative by

$\mathrm{P}_{\mathrm{iJ}}=1-\prod_{\mathrm{j} \in \mathrm{J}}\left(1-\mathrm{P}_{\mathrm{ij}}\right)$ or

$\mathrm{B}_{\mathrm{iJ}}=1-\Pi_{\mathrm{j} \in \mathrm{J}}\left(1-\mathrm{B}_{\mathrm{ij}}\right)$, respectively.

These last rules have an interesting foundation as they, under proper hypotheses (of maximal dependence for the rule of the maximum and of independence for the rule of the product), derive from the probabilities of being the best according to each criterion the probability of being the best according to at least one of them. Nevertheless, the sum is the easier form of making perceived the effect of increasing the probability of choice by joining the criteria.

\subsection{Capacities Construction Algorithm}

To maximize concentration, to each set of criteria $\mathbf{J}$ is assigned a capacity proportional to the maximum preference that such set assigns to some alternative. Its exact value will be obtained by scaling it so that capacity 1 is assigned to the set of all criteria.
Formally, the capacity assignment algorithm for the subset J will have the following two steps.

First, is computed, across all alternatives, the maximum of the joint preferences

$\mathrm{M}(\mathrm{J})=\max _{\mathrm{i} \in \mathrm{A}} \mathrm{P}_{\mathrm{i} J}$.

The capacity is achieved with a final standardization, which consists of dividing $\mathrm{M}(\mathrm{J})$ by the largest value $\mathrm{M}(\mathrm{S})$.

For the case of the $\mathrm{P}_{\mathrm{i} J}$ determined by the sum $\sum_{\mathrm{j} \in \mathrm{J}} \mathrm{P}_{\mathrm{ij}}$ this capacity is denoted by TPS. That means

$\operatorname{TPS}(J)=\max _{\mathrm{i} \in \mathrm{A}} \sum_{\mathrm{j} \in \mathrm{J}} \mathrm{P}_{\mathrm{ij}} / \max _{\mathrm{i} \in \mathrm{A}} \sum_{\mathrm{j} \in \mathrm{S}} \mathrm{P}_{\mathrm{ij}}$

for all $\mathrm{J}$.

Analogously are generated capacities TPM and TPP for the $\mathrm{P}_{\mathrm{iJ}}$ determined, respectively, by $\max _{i \in A} \max _{j \in J} P_{i j}$ and by $\max _{i \in A}\left[1-\Pi_{j \in J}(1-P i j)\right]$.

The same construction may be made starting with the $P_{i j}$ replaced by the $B_{i j}$. This will give rise to three different capacities: TBS, TBM and TBP.

In the case of a large number of criteria, a simplification that makes easier to explain the meaning of the interactions is produced limiting the application of these computations to sets with a limited number $\mathrm{L}$ of criteria, and assigning capacity 1 to all sets with more than L criteria. The cost of this simplification is a possible reduction of the importance assigned to those criteria with high interaction with other criteria.

For $\mathrm{L}=2$, this will give rise to six more capacities, 2PS, 2PM, 2PP, 2BS, 2BM and 2PP. For example, in the case of composition by addiction, equation (6) will be replaced by

$2 \mathrm{PS}(\mathrm{J})=1$

for all $\mathrm{J}$ with more than two criteria,

$2 \mathrm{PS}\left(\left\{\mathrm{j}_{1}, \mathrm{j}_{2}\right\}\right)=$

$\max _{i \in \mathrm{A}}\left(\mathrm{P}_{\mathrm{ij} 1}+\mathrm{P}_{\mathrm{ij} 2}\right) / \mathrm{max}_{\mathrm{i} \in \mathrm{A}, \mathrm{g} 1 \in \mathrm{S}, \mathrm{g} 2 \in \mathrm{S}}\left(\mathrm{P}_{\mathrm{ig} 1}+\mathrm{P}_{\mathrm{ig} 2}\right)$,

for every pair $\left(\mathrm{j}_{1}, \mathrm{j}_{2}\right)$ of different criteria in $\mathrm{S}$, and

$2 P S(j)=\max _{i \in A}\left(P_{i j}\right) / \max _{i \in A, g 1 \in S, g} 2 \in S\left(P_{i g 1}+P_{i g}\right)$

for all $\mathrm{j}$ in $\mathrm{S}$.

\subsection{The Choquet Integral}

The preference probabilities according to isolated criteria are combined by the Choquet integral. The Choquet integral replaces the weighted average when it is assumed that the presence of interaction between the criteria invalidates the use of compensatory addition.

For any function $\mathrm{x}=\left(\mathrm{x}_{1}, \ldots, \mathrm{x}_{\mathrm{T}}\right)$, with domain $\{1, \ldots, T\}$ and values in $R^{+}$, the Choquet integral of $\mathrm{x}$ in relation to a capacity $\mu$ on $\{1, \ldots, \mathrm{T}\}$ associates to $x$ the non-negative real number

$\mathrm{C}_{\mu}(\mathrm{x})=\sum\left(\mathrm{x}_{\tau(\mathrm{j})}-\mathrm{x}_{\tau(\mathrm{j}-1)}\right) \mu\{\tau(\mathrm{j}), \ldots, \tau(\mathrm{T})\}$,

for $\tau$ denoting a permutation of $\{1, \ldots, \mathrm{T}\}$ such that

$\mathrm{x}_{\tau(1)} \leq \mathrm{x}_{\tau(2)} \leq \ldots \leq \mathrm{x}_{\tau(\mathrm{T}-1)} \leq \mathrm{x}_{\tau(\mathrm{T})}$

and $\mathrm{x}_{\tau(0)}=0$. 
The Choquet integral is, equivalently, given by

$\mathrm{C}_{\mu}(\mathrm{x})=\sum \mathrm{x}_{\tau(\mathrm{j})}\left[\mu\left(\mathrm{Z}_{\tau(\mathrm{j})}\right)-\mu\left(\mathrm{Z}_{\tau(\mathrm{j}+1)}\right)\right]$

for $Z_{\tau(j)}=\{\tau(j), \ldots, \tau(T)\}$, for all $j$ from 1 to $T$, and $\mathrm{Z}_{\tau(\mathrm{T}+1)}=\phi$.

By choosing to compose by the Choquet integral to take interaction into account, one is also employing the principle of preference concentration. In fact, the Choquet integral gives more value to the highest preferences if they are obtained by applying a criterion with larger positive interaction with the others, and a lower value otherwise.

\section{Ranking Rules}

This section presents a list of composition rules, all employing the principle of preference concentration to deal with the presence of interaction. Twelve rules of composition are ordered according to the value of easy explanation. Each one is offered to be used in case of a tie in the choice by the previous ones.

The score of alternative $\mathrm{i}$ is, in the first six, obtained by calculating the Choquet integral of $P_{i}$ relatively to each of the six capacities derived from $\mathrm{P}_{\mathrm{i}}$ by the above procedures. The last six are obtained analogously by calculating the Choquet integral of $\mathrm{B}_{\mathrm{i}}$ relatively to capacities similarly generated from $\mathrm{B}_{\mathrm{i}}$. This implies assuming the transformation into probabilities of being the best as the less profitable source of complexity.

Inside each group of six, the three rules involving the computation of interaction for all sets of criteria come after those with fewer computations. This implies that the complexity involved in differentiating the sets of more criteria provides a more important differentiation than the transformation into probabilities of being the best, but even less important than the form of composition employed to join the evaluations by the different criteria.

Finally, inside each group of three with the same basic probabilities and the same number of interactions computed, considering that ordering from the easiest to explain to the more complex, addition comes before the maximum, and the product after it, the following order is obtained.

1. $\mathrm{P}_{\mathrm{i}}$ rating vectors and $2 \mathrm{PS}$ capability.

2. $P_{i}$ rating vectors and $2 \mathrm{PM}$ capacity.

3. $\mathrm{P}_{\mathrm{i}}$ rating vectors and $2 \mathrm{PP}$ capacity.

4. $P_{i}$ rating vectors and TPS capability.

5. $P_{i}$ rating vectors and TPM capability.

6. $P_{i}$ rating vectors and TPP capacity.

7. $B_{i}$ rating vectors and $2 \mathrm{BS}$ capacity.

8. $\mathrm{B}_{\mathrm{i}}$ rating vectors and $2 \mathrm{BM}$ capacity.

9. Bi rating vectors and $2 \mathrm{BP}$ capacity.
10. Bi rating vectors and TBS capacity.

11. Bi rating vectors and TBM capacity.

12. Bi rating vectors and TBP capacity.

\section{Case Study}

In this section, the composition rules above created are applied to choose one among seven alternatives of fuel supplier for an aviation enterprise.

The evaluations of the seven alternatives according to four criteria are given by [22]. The criteria are: 1) management and organization, 2) customer responsiveness, 3) transportation cost and 4) production cost. For the first two criteria there are three qualitative assessments of each alternative, and for the two last ones there are numerical values.

The distributions of preference probabilities according to the four criteria derived from the pairwise trichotomic comparisons of the assessments are given as column vectors in Table 1 . Thus, Table 1 reveals alternative 7 as the one with the lowest cost of production, alternative 1 as the one with the lowest cost of transport, alternative 4 as the one with the highest customer responsiveness and a tie between alternatives 1,2 and 6 regarding the first criterion. Alternative 1 , by the third criterion, and alternative 7 , by the fourth criterion, have the highest probability of preference by an isolate criterion, of, approximately, 0.2857 .

\section{Table 1. Initial ratings}

$\begin{array}{lllll}\text { Alt\Crit } & 1 & 2 & 3 & 4 \\ 1 & 0,1905 & 0,0794 & 0,2857 & 0,1429 \\ 2 & 0,1905 & 0,1508 & 0,1429 & 0,1905 \\ 3 & 0,1349 & 0,1746 & 0,2381 & 0,0476 \\ 4 & 0,1905 & 0,1825 & 0,0476 & 0,0952 \\ 5 & 0,0714 & 0,0238 & 0,0952 & 0,2381 \\ 6 & 0,1349 & 0,2143 & 0 & 0 \\ 7 & 0,0873 & 0,1746 & 0,1905 & 0,2857\end{array}$

Table 2 shows the probabilities of each alternative being the most preferred, still according to each criterion separately. These new probability distributions are obtained by attributing to each evaluation a standard beta pert distribution centered on the value given in Table 1. These results may be obtained applying the procedure PMax.Beta of the R package CPP [23].

It can be noted, comparing Tables 1 and 2, the increased ability to highlight in Table 2 the most preferred alternatives for the three criteria for which there is a unique most preferred alternative. The distance from the approximate probabilities of the most preferred alternative to the second goes from 
0.032 to 0.033 in the second criterion, from 0.048 to 0.061 in the third, and from 0.048 to 0.061 in the fourth.

Table 2. Probabilities of being the most preferred alternatives

$\begin{array}{lllll}\text { AltlCrit } & 1 & 2 & 3 & 4 \\ 1 & 0,184237 & 0,089415 & 0,280658 & 0,127057 \\ 2 & 0,184237 & 0,142985 & 0,127057 & 0,169066 \\ 3 & 0,132212 & 0,164885 & 0,219931 & 0,065751 \\ 4 & 0,184237 & 0,172673 & 0,065751 & 0,092933 \\ 5 & 0,086315 & 0,05877 & 0,092933 & 0,219931 \\ 6 & 0,132212 & 0,206388 & 0,044604 & 0,044604 \\ 7 & 0,096549 & 0,164885 & 0,169066 & 0,280658\end{array}$

Table 3 presents the capacities obtained from the probabilities of preference given in Table 1 , and Table 4 presents the capacities obtained from the probabilities of being the best given in Table 2. In the first three numeric columns of both tables are the capacities built assigning maximum capacity to all sets of more than 2 criteria, while in the last three are the capacities obtained by also calculating the effects of interactions among more than 2 criteria. From left to right, each of these groups of three capacities is ordered starting with the joint preference probability given by the sum, and following with the maximum. It is interesting to note the smaller variation in the second and penultimate columns, in both tables, due to the lesser discrimination offered by the maximum.

Table 3. Capacities derived from the initial ratings

$\begin{array}{lllllll}\text { CritlCapacity } & \text { 2SP } & \text { 2MP } & \text { 2PP } & \text { TSP } & \text { TMP } & \text { TPP } \\ 1 & 0.40 & 0.67 & 0.41 & 0.26 & 0.67 & 0.28 \\ 2 & 0.45 & 0.75 & 0.46 & 0.29 & 0.75 & 0.31 \\ 3 & 0.60 & 1 & 0.62 & 0.39 & 1 & 0.41 \\ 4 & 0.60 & 1 & 0.62 & 0.39 & 1 & 0.41 \\ 1 \& 2 & 0.78 & 0.75 & 0.79 & 0.51 & 0.75 & 0.53 \\ 1 \& 3 & 1 & 1 & 1 & 0.65 & 1 & 0.67 \\ 1 \& 4 & 0.80 & 1 & 0.80 & 0.52 & 1 & 0.54 \\ 2 \& 3 & 0.87 & 1 & 0.87 & 0.56 & 1 & 0.58 \\ 2 \& 4 & 0.97 & 1 & 0.97 & 0.62 & 1 & 0.65 \\ 3 \& 4 & 1 & 1 & 1 & 0.65 & 1 & 0.67 \\ 1 \& 2 \& 3 & 1 & 1 & 1 & 0.75 & 1 & 0.77 \\ 1 \& 2 \& 4 & 1 & 1 & 1 & 0.74 & 1 & 0.76 \\ 1 \& 3 \& 4 & 1 & 1 & 1 & 0.84 & 1 & 0.85 \\ 2 \& 3 \& 4 & 1 & 1 & 1 & 0.88 & 1 & 0.89\end{array}$

Table 4. Capacities derived from the probabilities of being the best

$\begin{array}{lllllll}\text { CritlCapacity } & \text { 2SB } & 2 \mathrm{MB} & \text { 2PB } & \text { TSB } & \text { TMB } & \text { TPB } \\ 1 & 0.40 & 0.66 & 0.45 & 0.26 & 0.66 & 0.34 \\ 2 & 0.44 & 0.74 & 0.50 & 0.29 & 0.74 & 0.38 \\ 3 & 0.60 & 1 & 0.68 & 0.39 & 1 & 0.51 \\ 4 & 0.60 & 1 & 0.68 & 0.39 & 1 & 0.51 \\ 1 \& 2 & 0.77 & 0.74 & 0.79 & 0.50 & 0.74 & 0.59 \\ 1 \& 3 & 1 & 1 & 1 & 0.65 & 1 & 0.75 \\ 1 \& 4 & 0.81 & 1 & 0.85 & 0.53 & 1 & 0.64 \\ 2 \& 3 & 0.83 & 1 & 0.84 & 0.54 & 1 & 0.63 \\ 2 \& 4 & 0.96 & 1 & 0.97 & 0.63 & 1 & 0.73 \\ 3 \& 4 & 0.97 & 1 & 0.97 & 0.63 & 1 & 0.73 \\ 1 \& 2 \& 3 & 1 & 1 & 1 & 0.78 & 1 & 0.85 \\ 1 \& 2 \& 4 & 1 & 1 & 1 & 0.76 & 1 & 0.83 \\ 1 \& 3 \& 4 & 1 & 1 & 1 & 0.83 & 1 & 0.89 \\ 2 \& 3 \& 4 & 1 & 1 & 1 & 0.86 & 1 & 0.91\end{array}$

A comparison of Tables 3 and 4 reveals the small effect of replacing the initial probabilities of preference by the probabilities of being the most preferred alternative. Except for some of the values of the capacities built involving the more elaborate use of the initial values in the calculations of the products, $2 \mathrm{PP}$ against $2 \mathrm{BP}$, and TPP against TBP, all differences are below 0.05 .

Finally, Table 5 presents the scores that determine the choice of the best alternative. The choice is based on the values in the first row, with a tie break by the values in the second, and so on. In this case, it is observed that the simplest rules, based on equal capacities for sets of more than two criteria, present a tie between alternatives 1 and 7. The choice of alternative 7 results from subsequently applying the TPS composition rule.

\section{Table 5. Scores by 12 composition rules}

$\begin{array}{llllllll}\text { Rule\Alt } & 1 & 2 & 3 & 4 & 5 & 6 & 7 \\ 1 & 0.25 & 0.18 & 0.21 & 0.17 & 0.18 & 0.14 & 0.25 \\ 2 & 0.29 & 0.19 & 0.24 & 0.17 & 0.24 & 0.16 & 0.29 \\ 3 & 0.25 & 0.18 & 0.21 & 0.17 & 0.18 & 0.14 & 0.25 \\ 4 & 0.2 & 0.17 & 0.16 & 0.13 & 0.13 & 0.09 & 0.21 \\ 5 & 0.29 & 0.19 & 0.24 & 0.17 & 0.24 & 0.16 & 0.29 \\ 6 & 0.2 & 0.17 & 0.16 & 0.13 & 0.14 & 0.1 & 0.22 \\ 7 & 0.25 & 0.18 & 0.21 & 0.17 & 0.18 & 0.14 & 0.25 \\ 8 & 0.29 & 0.19 & 0.24 & 0.16 & 0.24 & 0.16 & 0.29 \\ 9 & 0.26 & 0.18 & 0.21 & 0.17 & 0.19 & 0.15 & 0.25 \\ 10 & 0.20 & 0.17 & 0.16 & 0.13 & 0.13 & 0.09 & 0.21 \\ 11 & 0.29 & 0.19 & 0.24 & 0.16 & 0.24 & 0.16 & 0.29 \\ 12 & 0.22 & 0.17 & 0.18 & 0.14 & 0.16 & 0.11 & 0.23\end{array}$


This result coincides with that obtained in [22]. The scores in Table 5, however, draw attention to the close proximity between the evaluations of alternatives 1 and 7 , with scores considerably distinct from those of the other alternatives.

\section{Conclusion}

A methodology for agile decision is developed here and applied to a supplier selection case. Several features of the system ensure its speed and efficiency. First, data collection requires only pairwise trichotomic comparison, which reduces the possibility of errors and the effect of the imprecision of the evaluations. The composition of the criteria is automatic with the application of preference concentration to take into account interaction. Finally, a conclusive decision is guaranteed by ordering a set of similar variants of the decision rule.

To validate the model, it can be checked against more complex specifications. The trichotomic approach may be replaced with admitting more than three values. Each evaluator may also be advised to apply a particular pattern in the differentiation of the alternatives.

Artificial intelligence can be used to choose between models with different sets of criteria. Computer simulation can be applied to generate preference probability distributions for the alternatives.

Sampling for the direct determination of the importance of the criteria may also be employed. To preserve agility, the experts may be asked to provide only trichotomic evaluations of the criteria, to be combined additively.

The weights so generated may instead be used in an improvement of the last stage. The Choquet integral may be applied to the matrix of the $\mathrm{P}_{\mathrm{ij}}$ modified by reducing the values of the columns of vectors of evaluations proportionally to the weights obtained for the respective criteria.

\section{References:}

[1] Keeney, R. \& Raiffa, H. Decisions with Multiple Objectives: Preferences and Value Tradeoffs. Wiley, 1976.

[2] Bronner, R. Decision making under time pressure. Lexington Books, 1982.

[3] Eisenhardt, K. Making fast strategic decisions in high-velocity environments. Academy of
Management Journal, Vol. 32, No. 3, 1989, pp. 543-576.

[4] Cohen, M., Freeman, J. \& Wolf, S. Metarecognition in time-stressed decision making: recognizing, critiquing and correcting. Human Factors, Vol. 38, No. 2, 1996 pp. 206-219.

[5] Lehner, P., Seyed-Solorforough, M., O'Connor, M., Sak, S. \& Mullin, T. Cognitive biases and time stress in team decision making. IEEE Transactions on Systems, Man, and Cybernetics, Vol. 27, No. 5,1997, pp. 698-703.

[6] Fisher, C., Chengalur-Smith, I. \& Ballou, D. The Impact of Experience and Time on the Use of Data Quality Information in Decision Making. Information Systems Research, Vol. 14, No. 2, 2003, pp. 170-188.

[7] Walk, S. A new fast reliable filtering method for multicriteria decision making. Management Decision, Vol. 49, No. 5, 2011, pp. 810-822.

[8] Sant'Anna, A. P., Nogueira, H. D. \& Rabelo, L. M. Probabilistic Composition for Fast Group Decisions. Brazilian Journal of Operations \& Production Management, Vol. 8, No 1, 2011, pp. 65-82.

[9] Garcia, P. A. A. \& Sant'Anna, A. P. Vendor and Logistics Provider Selection in the Construction Sector: a Probabilistic Preferences Composition Approach. Pesquisa Operacional, Vol. 35, No. 2, 2015, pp. 170188.

[10] Dursun, M \& Ogunchu, O. Agile Supplier Evaluation Using Hierarchical TOPSIS Method. WSEAS Transactions on Information Science and Applications, Vol. 18, 2021, pp.12-19.

[11] Hwang, C. L. \& Yoon, K. Multiple attribute decision making: methods and applications. Berlin: Springer, 1981.

[12] Ateş, N.Y., Çevik S., Kahraman, C., Gülbay, M. \& Erdoğan, S. A. Multi attribute performance evaluation using a hierarchical fuzzy TOPSIS method, studies in fuzziness and soft computing. Fuzzy Applications in Industrial Engineering, Vol. 201, 2006, pp. 537-572.

[13] Chen, C. T., Lin, C. T. \& Huang, S. F. A fuzzy approach for supplier evaluation and selection in supply chain management, International Journal of Production Economics, Vol.102, No.2, 2006, pp.289-301. 
[14] Sant'Anna, A. P. Probabilistic Composition of Preferences: Theory and Applications. Springer, 2015.

[15] Deng, Ju-Long Control problems of grey systems. Systems \& Control Letters. Vol. 1, No. 5, 1982, pp. 288-294.

[16] Li, W., Wang, Y. \& Chen, A. Grey relational evaluation on vendor selection based on ebusiness. Annals of the International Conference on Electronic Commerce and Security, 2008, pp. 509-513.

[17] Li, W. Using grey multipurpose decision system for vendor selection based on ebusiness. Proceedings of the 2009 Information Technology and Computer Science Conference, 2009, pp. 27-30.

[18] Yang, Y. \& Wu, L. Improved grey correlative method for green vendor selection in coal group. International Conference on Internet Technology and Applications, 2010, pp. 1-4.

[19] Rajesh, R. \& Ravi, V. Supplier selection in resilient supply chain: a grey relational approach. Journal of Cleaner Production, Vol. 86, 2015, pp. 343-359.

[20] Choquet, G. Theory of capacities. Annales de l'Institut Fourier, Vol. 5, 1953, 131-295.

[21] Sant'Anna, A. P. \& Sant'Anna, J. L. A Principle of Preference Concentration applied to the unsupervised evaluation of the importance of multiple criteria. Pesquisa Operacional, Vol. 39, No. 2, 2019, pp. 317338.

[22] Dursun, M., Goker, N \& Ogunclu, O. A Fuzzy Group Decision Making Approach for Agility Evaluation in Aviation Industry. Proceedings of the World Congress on Engineering 2017 Vol. II, pp. 688-691.

[23] Gavião, L. O., Sant'Anna, A. P., Lima, G. B. A. \& Garcia, P. A. de A. CPP: Composition of Probabilistic Preferences. $R$ package https://cran.rproject.org/package=CPP.

2018.

\section{Creative Commons Attribution \\ License 4.0 (Attribution 4.0 International , CC BY 4.0)}

This article is published under the terms of the Creative Commons Attribution License 4.0 https://creativecommons.org/licenses/by/4.0/deed.en US 